\title{
E-Vanguard for Emergency - A Wireless System for Rescue and Healthcare
}

\author{
Shun-Chuan Chen, Hsuan-Wei Chen, Ang Lee, Kuo-Hao Chao, Yu-Cheng Huang and Feipei Lai \\ College of Electrical Engineering and Computer Science, National Taiwan University \\ Taipei 106, Taiwan \\ hypno@speech.ee.ntu.edu.tw
}

\begin{abstract}
E-Vanguard for Emergency, called hereafter the $E V E$, provides a system that helps the rescue and first-aid work in the present rescue operation. The EVE consists of rescuers equipped with wearable PDAs, medics with laptops plus GPS, a rescue command center controlled by the leaders of the rescue and medical teams, and a monitor subsystem including physical sensors, such as an oximeter and an electro-cardiograph, provided with wireless transmission for the injured. Each part communicates with all the others using Bluetooth. The EVE improves the rescue work by monitoring rescuers' physical conditions and the environment temperature to give an early warning. It also helps first-aid work by providing medics with real-time information about the injured and having in hand the medics' positions for efficient allocation of medical resources. In addition, the system collects instant medical information of the injured through sensors and transmits the data to the nearby medics or rescuers, which are further relayed back to the rescue command post. The unique feature of Bluetooth is exploited by the EVE system to construct a Bluetooth relay network, a Scatternet.
\end{abstract}

\section{INTRODUCTION}

Frequently there are disasters all over the world - fires, earthquakes, or even some unexpected shocking catastrophes. Hence people injured, or even died, which we were unwilling to see. According to the statistics from the Taiwan Fire Administration and the emergency center of the National Taiwan University Hospital, many people died in accidents because the hospitals neither had enough first-hand information nor had a full-scale rescue system. Even unfortunately, many rescuers lost their lives during the rescue operation. This was what motivated us to develop a rescue system to assist the present one.

The EVE system collects instant medical information of the injured through sensors and transmits the data to the nearby medics or rescuers, which are further relayed back to the rescue command post. The most important progress EVE has made is the integration of various technologies, including digital signal processing for medical sensors, network technologies such as GPS and Bluetooth, and an embedded system for wearable PDAs, to form a wireless rescue network.

The system is designed with a server-client framework. The server plays the role as a dispatching control center that manages all rescue operation. Rescuers and medics, the client, are responsible for communicating with the server through Bluetooth and taking appropriate action. In addition, the system provides a mobile device of physical sensors for rescuers and the rescued, including an electro-cardiograph and an oximeter that can detect and send back the life conditions of the injured as soon as possible. A thermometer sensor is further built in the device to monitor the environment for the rescuers to give a timely warning. The Global Positioning System (GPS) is also applied for tracking the positions of the medics. It increases the management flexibility and enables an efficient resource allocation. The EVE uses Bluetooth to provide a wireless interface among users on different platforms. It exploits the unique feature of Bluetooth to construct a relay Bluetooth Scatternet. Therefore, the EVE system can expand the scope of the rescue operation, while the communication among all members remains fast and reliable. The overall system diagram is shown in Fig. 1.

In order to be an effective rescue system, we planned to design a system that is real-time, portable, and user friendly. In this paper, the experiments show satisfactory results.

The rest of the paper is organized as follows. Component technologies are discussed in detail in Section II. In Section III, the overall system flow is given. The experiment results and validations are presented in Section IV. Finally, the conclusion and future work are in Section V.

\section{COMPONENT TECHNOLOGIES}

\section{A. Network Technology Integration}

In the EVE system, we established a communication mechanism to manage all the information interchange. New applications can be added into the system as long as they are designed using the following mechanism with Bluetooth. The network constructed from the communication mechanism, called the EVE Net, is shown in Fig. 2.

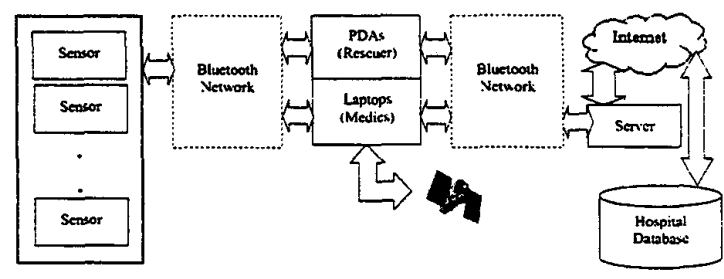

Figure 1. The EVE system. 


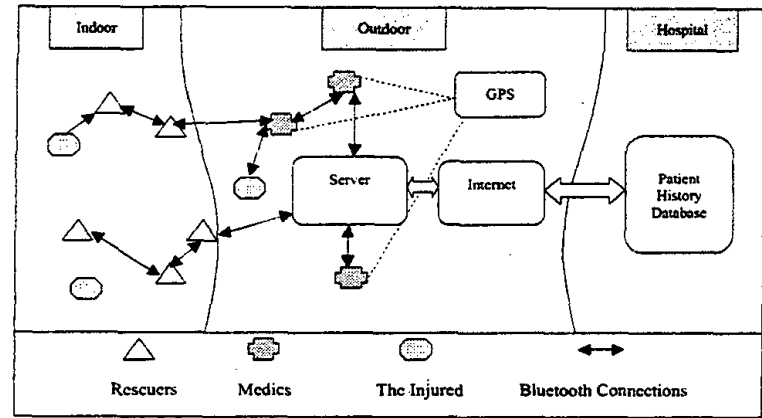

Figure 2. The EVE Net.

We used three kinds of network techniques to implement the EVE Net.

\section{A.1. Bluetooth}

Communication among the sensors, which are attached to the injured, the medics, the rescuers, and the server, is a pure Bluetooth network. This network was designed to be hierarchical, which is shown in Fig. 3.

According to the Bluetooth Specification Version 1.1 [1], we implemented the $\mathrm{HCl}$ layer, the Logical Link Control Adaptation Protocol (L2CAP) layer, the RFCOMM layer, and the Service Discovery Protocol (SDP), as shown in Fig. 4.

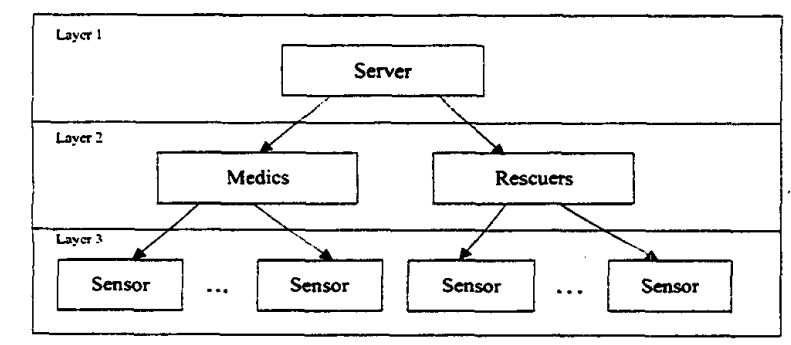

Figure 3. The hicrarchy of the Bluetooth network.

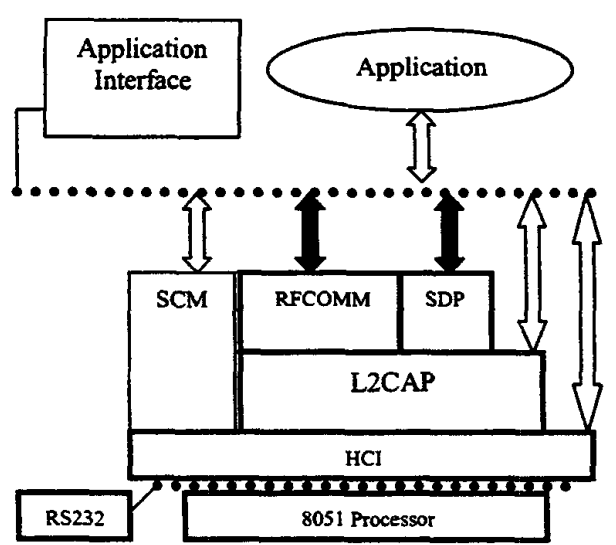

Figure 4. Bluetooth stack: sensors.
We built the Bluetooth module connection for the sensors, so the sensors can connect to any standard Bluetooth module, such as XIRCOM Bluetooth PC-card. The serial port is used to transfer data with transmission rate up to $115200 \mathrm{bps}$. In addition, in order to increase the compatibility of sensors, we implemented the SDP, which makes it possible for all Bluetooth devices with SDP to be connected and to discover which services are available in the applications.

One of the superior features of the EVE system is the implementation of Bluetooth in various scales, including the Piconet and the Scatternet [2]. The multipoint Bluetooth modules on PDAs and laptops are able to simultaneously interconnect up to 8 transceivers in a Piconet over a short range. However, by operating among several Piconets in a close proximity, the Bluetooth devices can frequently move from one Piconet to another, which effectively overcomes the 8device constraint. In the EVE system, there are two or even more interconnected Piconets forming a Scatternet, taking advantage of their capability to operate within the vicinity of each other with different hopping sequences. By having several small groups of Bluetooth devices connected with each other in the same area, we maintain a wireless network with large rescue coverage. Furthermore, we use a master relay to build up the Bluetooth relay. When a new device from the rescuers or medics is inquiring the server, the server will check the address of the device. A relay node device will immediately exchange the data. On the contrary, the connection is dropped once the server finds out the device address is incorrect. A list of Bluetooth addresses, which correspond to the name/ID of a person, is recorded for the present connection, allowing the server to identify the clients. We used a modified Tree Scatternet Formation algorithm [3] to efficiently connect nodes in a tree structure that simplifies packet routing and scheduling.

Furthermore, a Bluetooth stack security was implemented. The EVE system has two different layer-to-layer Bluetooth networks. Therefore, we used two different group-based securities. Any Bluetooth node that wants to participate in the system needs to conform to its own security format. The data are encrypted with their own UID and LayerID. An example of UID and LayerID encryption is given in Fig. 5. In this way, the data are encrypted, and other Bluetooth devices, which do not belong to the EVE system, will not intercept them.

LayerID1 $=[\{28 \mathrm{~B} 0 \mathrm{C} 88 \mathrm{~B}-7 \mathrm{CA} 1-4 \mathrm{DB} 0-\mathrm{BAB} 2-9 \mathrm{E} 9 \mathrm{~F} 5481 \mathrm{~F} 4 \mathrm{C} 1\}]$

$\left.\mathrm{UID} 1=[\{C 7708690-9581-4 \mathrm{~B} 70-8 \mathrm{D} 6 \mathrm{C}-6131 \mathrm{D} 9543 \mathrm{CBD}\}\}^{\prime}\right]$

UID2 $=\left[{ }^{\prime}\{\right.$ A67D3D16-32F0-452]-8E0F-F3C8069EC487 $\left.\}\right]$

UID3 $=\left[{ }^{\prime}\{\right.$ ED8460D7-29E6-40B0-8650-5891CBD9DA35 $\left.\}\right]$

LayerID2 $=[\{$ D4425370-5A7F-4099-B681-EDCD74ADB475\}']

UIDl $=[\{7$ F4808C9-F899-4A7F-BE3B-F44421EE268E $\}]$

UID2 $=\left[{ }^{\prime}\{9 A 05949 F-382 C-4013-9 E 76-295 D C 85 D C D 7 E\} '\right]$

Figure 5. A Bluetooth security example.

A.2. GPS

A GPS module is installed in every laptop held by outdoor medics. It calculates the medics' positions using the 
information from satellites, and sends them to the server by means of Bluetooth.

The GPS module (EverMore GM-X205), shown in Fig. 6, supports the NXMEA-0183 output format defined by the National Marine Electronic Association (NMEA). We mainly use the GGA-GPS Fix Data, which is one of NMEA messages.

The GPS format is in the following.

SGPGGA. hhmmss. dddmm.mmmm, a, dddmm.mmmm, a, $x, x x ., x . x, x . x$, M.,M, x.xxxx*GS

The time, latitude, and longitude information are combined to calculate the rescuers' positions. Then, the message is sent to the server from the laptops of the medics, whose positions can be displayed on the electronic map of the server.

\section{A.3. TCP/IP or GSM}

The server uses traditional Internet protocols, TCP/IP, or GSM to obtain information from hospitals, such as the patient's medical histories, to help the rescue operation. We use Microsoft Visual .Net Web Service [4] to implement the links to hospitals.

\section{B. A Real-time Embedded System}

Wearable Personal Digital Assistants (PDAs) are used for rescuers in the rescue operation. By means of Bluetooth, a PDA can receive messages from the monitoring sensors attached to the injured, analyze the signals, and send them over to the server. At the same time, it also receives all the rescuerelated information from the server. The user interface of PDAs carried by the rescuers is shown in Fig. 7.

Each button on the PDA can be accessed with fingertips. There are several functions provided. First, an emergency button is implemented in the PDA, which enables the rescuers to send an SOS message, either for himself or for the injured person just found. Second, when the blood oxygen concentration is below a certain threshold, the PDA will give a warning by making the "Blood $\mathrm{O}_{2}$ " field red and a "beep" sound.

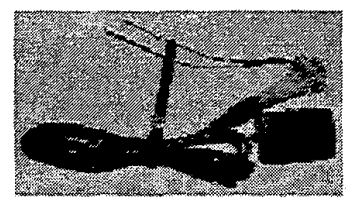

Figure 6. The GPS module.

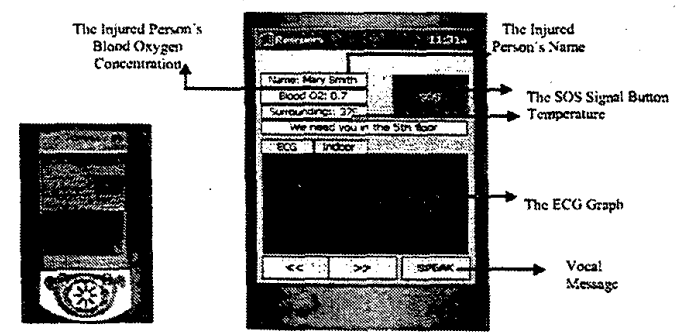

Figure 7. The PDA user interface.
Third, in an emergent situation, a vocal control may be a better choice than a text-based message. Finally, when the PDA receives IR-channel and R-channel signals via Bluetooth from the monitor, it will calculate the arterial oxygen saturation, $\mathrm{SaO}_{2}$, according to the IR- and $\mathrm{R}$ - channel waveforms. The definition of $\mathrm{SaO}_{2}$ is as follows.

$$
\mathrm{SaO}_{2}=\mathrm{C}_{\mathrm{HbO}} /\left(\mathrm{C}_{\mathrm{HbO}_{2}}+\mathrm{C}_{\mathrm{Hb}}\right)
$$

where $\mathrm{C}_{\mathrm{HbO}}=$ concentration of oxyhemoglobin $\left(\mathrm{HbO}_{2}\right)$, and $\mathrm{C}_{\mathrm{Hb}}=$ concentration of hemoglobin $(\mathrm{Hb})$

The $\mathrm{SaO}_{2}$ is determined by the $\mathrm{AC}$ and $\mathrm{DC}$ signals of both IR and $R$ channels. Based on the Beer-Lanber Law, R/IR ratio is defined as

$$
\frac{R}{I R}=\frac{\log \left(I_{(A C+D C) R} / I_{D C . R}\right)}{\log \left(I_{(A C+D C), R} / I_{D C . R}\right)}
$$

where $I_{(A C+D C) R}, I_{D C, R}, I_{(A C+D C) / R .}, I_{D C, R}$ are the intensity of red and infrared lights. $I_{(A C+D C}$ corresponds to the crest, and $I_{D C}$ corresponds to the trough of the waveform of $R$ and IR channel signals, respectively. The ratio of the normalized AC signal (2) at two wavelengths (R/IR) is related to $\mathrm{SaO}_{2}$ by the following well-known equation [5]:

$$
\mathrm{SaO}_{2}=\frac{\varepsilon_{I R}^{H b} \times(R / I R)-\varepsilon_{R}^{H b}}{\left(\varepsilon_{I R}^{H b}-\varepsilon_{I R}^{H b O_{2}}\right) \times(R / I R)+\left(\varepsilon_{I R}^{H b O_{2}}-\varepsilon_{R}^{H b}\right)}
$$

where $\varepsilon(\mathrm{Hb})$ and $\varepsilon\left(\mathrm{HbO}_{2}\right)$ are the absorption rates of $\mathrm{Hb}$ and $\mathrm{HbO}_{2}$. Now we derive crests and troughs of $\mathrm{R}$ and IR signals by a biological signal detection algorithm [6][7]. Then we can get $I_{(A C+D C) R}, I_{D C, R}, I_{(A C+D C) R R}, I_{D C, J R}, \mathrm{R} / \mathrm{IR}, \mathrm{SaO}_{2}$, sequentially.

\section{Digital Signal Processing}

The EVE Monitor Kit consists of the micro-processing circuits with a Bluetooth module and several sensor modules to communicate with PDAs/laptops via Bluetooth. The monitor kit, which weights only about $500 \mathrm{~g}$ and is shown in Fig. 8, is designed to be wearable for the injured and the rescuers.

The hardware system diagram is shown in Fig. 9. An 8051CPU-based system is used to integrate the sensor module and Bluetooth. The picture of the EVE Monitor Kit is shown in Fig. 10.

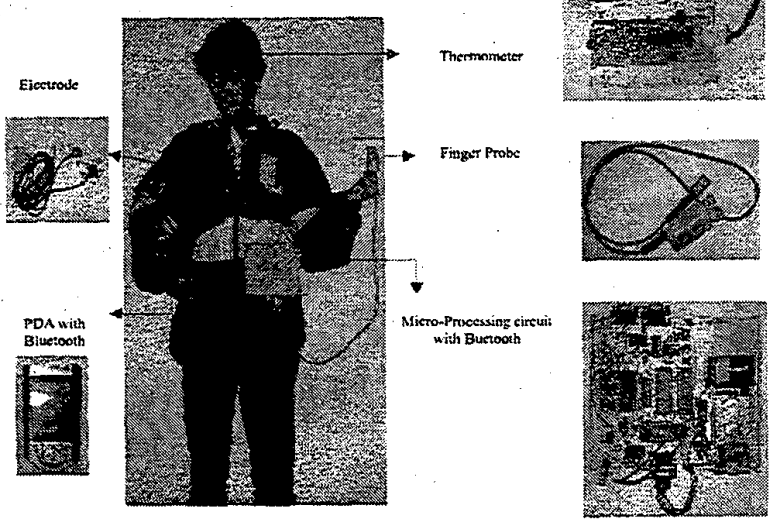

Figure 8. The equipments of a rescucr. 
In addition, in the EVE system, an RS-232 port is used to communicate with the Bluetooth Kit. We applied Time Division Pulse Modulation (TDPM) technique to enable the multi-channels. Three sensor modules were implemented to do the physical monitoring, including an electro-cardiograph, an oximeter, and an outdoor thermometer. Any new specific sensor module using RS-232 can be integrated into the EVE Monitor Kit easily.

\section{C.1. An Electro-Cardiograph (ECG)}

The ECG circuit is used to calculate the pulse of the injured or rescuers. Three electrodes are attached on the skin of the injured person's designated area. One is placed near the right collarbone, another is placed near the left abdomen, and the other is placed on the right leg to be signal ground, which measures the voltage $V_{A}, V_{B}$, and $G N D$, respectively. In addition, a chip AD620 is used to overcome the noise problem. Finally, through a $4^{\text {th }}$-order butterworth filter (cut-off frequency $=30 \mathrm{~Hz}$ ), the signal is sent to the ADC. The ECG block diagram is shown in Fig. 11.

\section{C.2. An Oximeter}

The oximeter measures the degree of oxygenation in artery, which is a critical index to determine whether the injured person is in danger. The arterial oxygen saturation, $\mathrm{SaO}_{2}$, can be derived from the red and infrared photo-plethysmograph signals. The oximeter is based on a "noninvasive" optical detection method and the $\mathrm{SaO}_{2}$ model. Its block diagram is shown in Fig. 12.

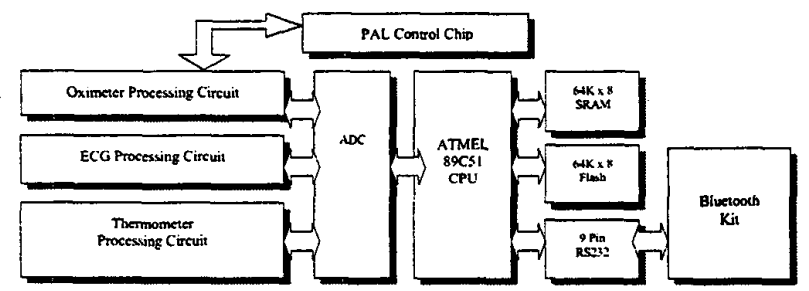

Figure 9. Hardware system diagram.

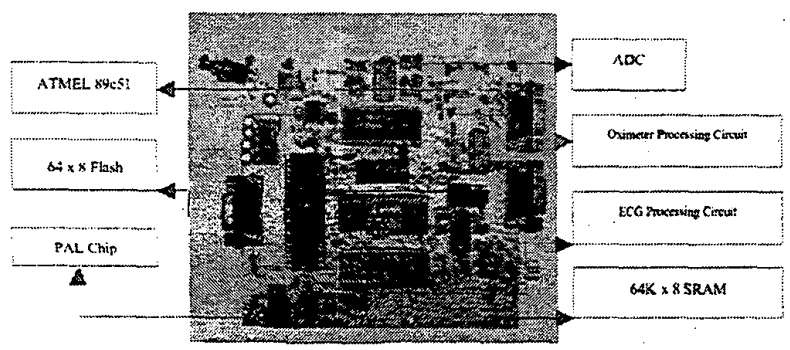

Figure 10. The EVE Monitor Kit

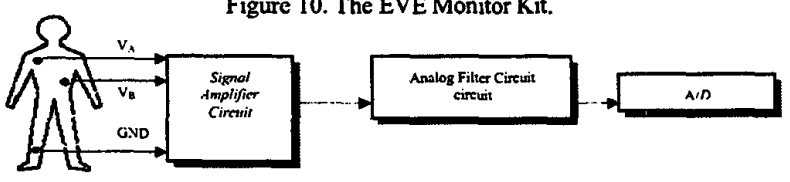

Figure 11. The ECG block diagram.

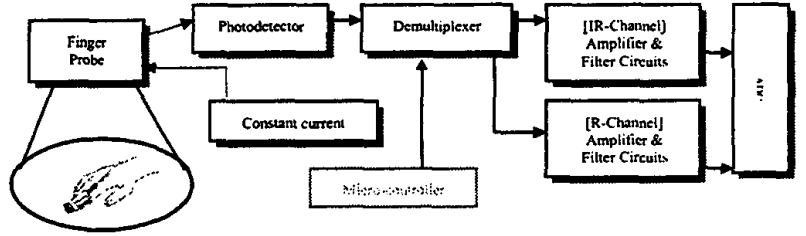

Figure 12. The oximeter block diagram.

We used the DS-100A sensor that has two LEDs and a photodiode on opposite sides of the fingertip (vascular bed). The light emitted from LEDs is absorbed, reflected, or diffusively scattered by tissues and blood when passing through the finger, and then collected by the photodiode. Due to the different absorption properties of reduced oxyhemoglobin $\left(\mathrm{HbO}_{2}\right)$ and hemoglobin $(\mathrm{Hb})$, the intensities of the red and infrared transmission lights become different after passing through a fingertip. Thus, the photodiode is used to transfer the light intensity into current intensity.

\section{C.3. A Thermometer}

To get the temperature of some dangerous environment is very important. In the EVE system, a temperature transducer AD590, which nominal current output is $298.2 \mu \mathrm{A}$ at $+25^{\circ} \mathrm{C}$ (298.2K) and temperature coefficient is $+1 \mu \mathrm{A} / \mathrm{K}$, is used to give an early waming to the rescuers. After converting the output current of AD590 into a voltage signal, we change the temperature coefficient into $+100 \mathrm{mV} / \mathrm{K}$ by using the amplifier circuit and then send the signal to the ADC.

\section{THE OVERALL SYSTEM FLOW}

In this part, we will discuss the overall software implementations in detail. First, let's see the block diagram of the software architecture in Fig. 13.

Medics carry laptops with the GPS, communicating with the server via Bluetooth. In this way, they can find the needed information of the injured and do the best resource allocation in an emergency. Fig. 14 shows the user interface of the laptop.

We allowed the laptops to connect with the patient-history subsystem in the hospital via the command center. If a medic knows the injured person's identity, the connection with the patient-history subsystem can offer him helpful information.

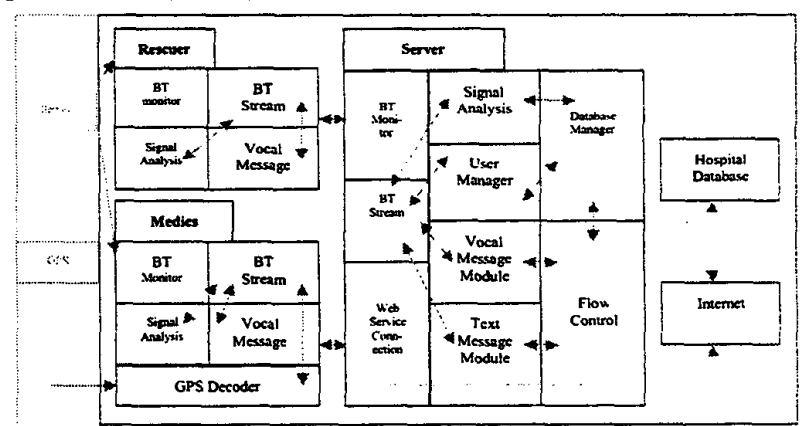

Figure 13. The software architecturc. 
The GPS module is installed in the laptops for the medics. Using the GPS, the command center can keep track of the movements of the medics, which enables an efficient resource management.

Now we will take a closer look at the server. The server is the central unit of the EVE system. It serves as a key position for the information communications. A sensor can only send data to its nearest PDAs or laptops, and finally communicate with the server via the EVE Net. The vocal message is transmitted and received in real-time and uses almost more than half of the bandwidth. All of the flow control work is done behind the scene, which is transparent to the server operator.

One of the features of the EVE server is its friendly GUI. Fig. 15 shown below is the main menu of the EVE server. The server is responsible for most of the central work. On the lefthand side of the screen are the data grids of rescuers, medics, and the injured. The data shown on the grid can be synchronized with the sensors. On the right-hand side shows the GPS map. The position of the selected person on the maps with GPS will be specifically shown when the corresponding row is clicked. By double clicking it, the rescue team can see the real time status relative to the selected person. Using GPS data from the medics, the server is able to identify the position of the medics on the electronic map.

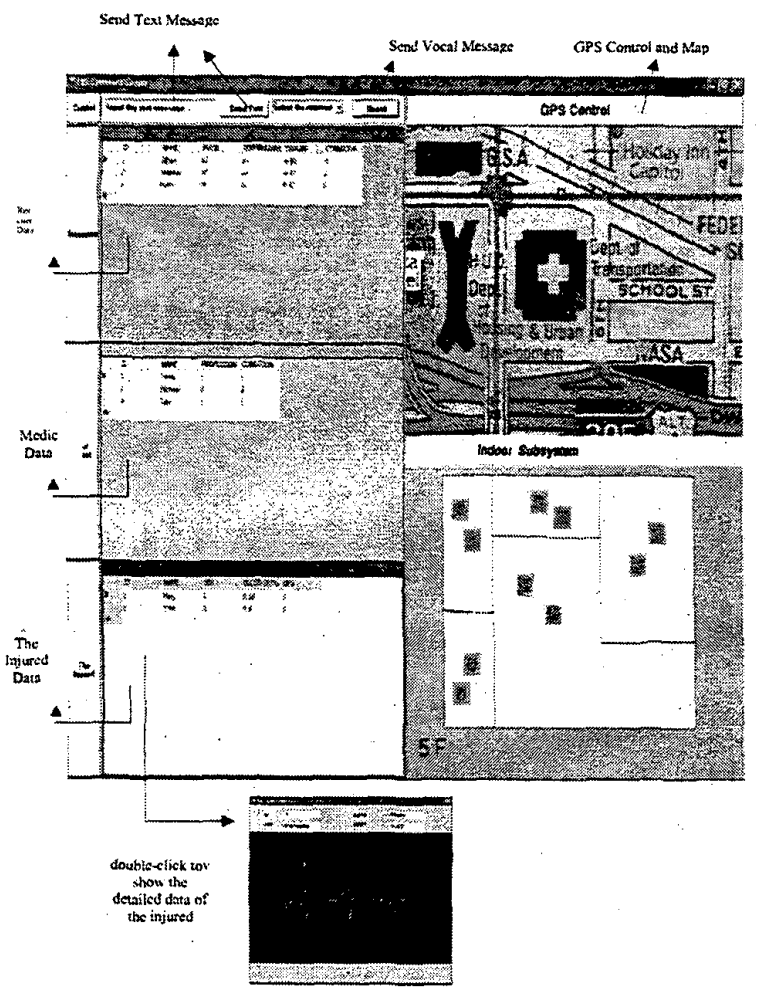

Figure 15. The server user interface.

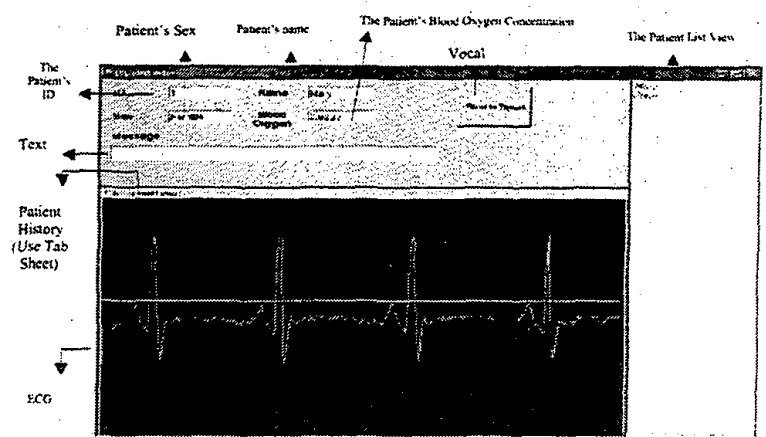

Figure 14. Laptop user interface.

When a medic moves, the map will zoom accordingly and the position points will shift dynamically. Integrating with the GPS enables the server to have better control over the medics. In addition, by connecting to a central database server for the patient history, the server can retrieve the patient history of the injured person once he knows the person's identity.

\section{EXPERIMENTS AND VALIDATIONS}

We measured the connection time between 10-meter Bluetooth module and our devices, including the server, PDAs, and laptops, with varying distances in Table $I$. The connection from sensors to PDAs must be switched to laptops after the injured has been taken out and handed over to the medics. The passoff between the rescuer (master) and the medics (another master) takes little time, so the data loss is tolerable.

The EVE also guaranteed a real-time transmission feature. We did the test by dividing the devices into two groups. One was still, and the other was moving. Because the entire devices in our system move very often, we tried to find out the transmission rate in reality of our system. The following tables show the test results.

TABLE I

The CONNECTION Setup Time

\begin{tabular}{|c|c|c|}
\hline \multirow{2}{*}{ Distance } & \multicolumn{2}{|c|}{ Connection Time (sec) } \\
\cline { 2 - 3 } & Sensor to Laptop & Sensor to PDA \\
\hline$<1 \mathrm{~m}$ & 3.90 & 3.78 \\
\hline $1 \mathrm{~m} \sim 3 \mathrm{~m}$ & 4.18 & 4.12 \\
\hline $3 \mathrm{~m} \sim 6 \mathrm{~m}$ & 5.75 & 5.81 \\
\hline $6 \mathrm{~m} \sim 9 \mathrm{~m}$ & 8.91 & 8.95 \\
\hline Average & 5.68 & 5.67 \\
\hline
\end{tabular}

TABLE II

TRANSmission Rate of STILL DeVICES (PER ChanNel UP TO 115200 bPS)

\begin{tabular}{|c|c|c|}
\hline \multirow{2}{*}{ Distance } & \multicolumn{2}{|c|}{ Transmission Rate } \\
\cline { 2 - 3 } & No Obstacle & Separated by a Wall \\
\hline $0 \mathrm{~m} \sim 5 \mathrm{~m}$ & $>50 \mathrm{Kbps}$ & $47 \mathrm{Kbps}$ \\
\hline $5 \mathrm{~m} \sim 10 \mathrm{~m}$ & $>50 \mathrm{Kbps}$ & $40 \mathrm{Kbps}$ \\
\hline
\end{tabular}


TABLE III

TRANSMISSION RATE OF MOVING DEVICES (per channel up to $115200 \mathrm{bps}$ )

\begin{tabular}{|c|c|c|}
\hline \multirow{2}{*}{ Distance } & \multicolumn{2}{|c|}{ Transmission Rate } \\
\cline { 2 - 3 } & No Obstacle & Separated by a Wall \\
\hline $0 \mathrm{~m} \sim 5 \mathrm{~m}$ & $>50 \mathrm{Kbps}$ & $42 \mathrm{Kbps}$ \\
\hline $5 \mathrm{~m} \sim 10 \mathrm{~m}$ & $>40 \mathrm{Kbps}$ & $36.6 \mathrm{Kbps}$ \\
\hline
\end{tabular}

TABLE IV

SENSOR DATA FORMAT

\begin{tabular}{|c|c|c|}
\hline Header & Data & Bluetooth Header \\
\hline 2 bytes & 4 bytes & 7 bytes \\
\hline \multicolumn{3}{|c|}{$\begin{array}{l}\text { Total rate: } 13 \text { bytes } / 7.5 \mathrm{~ms}=1.73 \mathrm{Kbps} \\
\text { (The sensor sends a data packet every } 7.5 \text { milliseconds, } \\
\text { which is measured with a logic analyzer.) }\end{array}$} \\
\hline
\end{tabular}

According to Table II and Table III, we know the transmission rate of the moving device is greater than transmission rate of the sensor data. We also evaluated how many sensors one Bluetooth channel can support. Because of the limitation of the number of Bluetooth devices, we did the test by duplicating the data transmitted by one sensor. Fig. 16 represents the testing environment.

In this way, we can duplicate the sensor's data and transmit them together in one Bluetooth channel. Therefore, we evaluate the number of the sensors one channel of the relay nodes can support. The evaluation result is shown in Table V. We also leamed that a Bluetooth channel can support at least 12 sensors in the EVE system. In addition, the power consumption of EVE Monitor was also measured. One $9 \mathrm{~V}$ battery can last for 80 minutes while all sensors are running. The result is shown in Table VI.

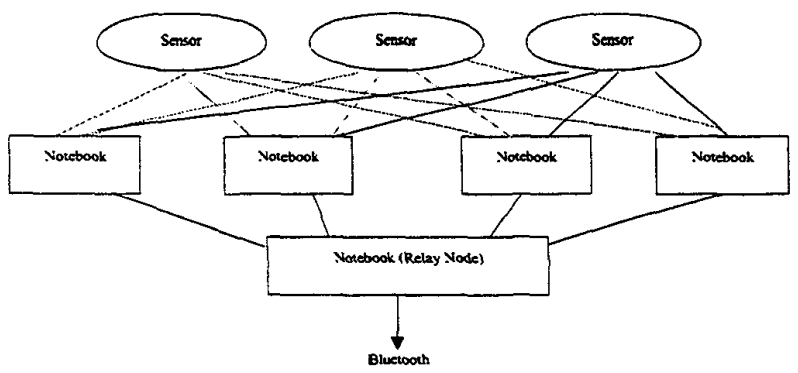

Figure 16. The evaluation environment of Bluetooth channel maximum loading.

TABLE V

Evaluation Result of Bluetooth Channel LoAding.

\begin{tabular}{|c|c|}
\hline Number of Sensors & Support ? \\
\hline 3 & Yes \\
\hline 6 & Yes \\
\hline 9 & Yes \\
\hline 12 & Yes \\
\hline
\end{tabular}

TALBE VI

POWER CONSUMPTION OF EVE MONITOR.

\begin{tabular}{|c|c|}
\hline $\begin{array}{c}\text { Bluetooth Connection } \\
\text { Setup }\end{array}$ & $\begin{array}{c}\text { Connection } \\
\text { Established }\end{array}$ \\
\hline $1.5 \mathrm{~W}$ & $0.9 \mathrm{~W}$ \\
\hline
\end{tabular}

According to our experiment results, we know that when Bluetooth devices are moving, the transmission rate will drop down. Now we use the $10-\mathrm{m}$ Bluetooth module to build up the system. If a large disaster area is encountered, we have to use 100-m Bluetooth devices. Then, the collision probability will increase, and the data transmission rate will drop down. Therefore, we have to reduce the sensor's transmission rate (1.7Kbps). If the transmission rate is lower than some threshold, the Eve system has the ability to disable some unused Bluetooth devices. Consequently, we can guarantee that the system can provide the channel to transfer the most important data.

We did some experiments on our physical sensors. From the testing, we found that the DC of the ECG data we got from the monitor would drift when rescuers or patients moved. This situation is shown in Fig. 17 and Fig. 18. It is because the DC voltage, $V_{A}-V_{B}$, changes. (see p. 4) We improved this by decreasing the Gain before the Analog Filter Circuit and increasing the Gain after the AFC. In addition, we made the electrodes closer to the skin to avoid the friction between them.

In addition, there is a trade-off between accuracy and sampling rate in terms of an oximeter. Resolution is proportional to the accuracy. However, if high accuracy is desired, more data have to be processed by micro-processor and transmitted by the Bluetooth kit, which decreases the speed of the overall system, and the sampling rate will fall naturally.

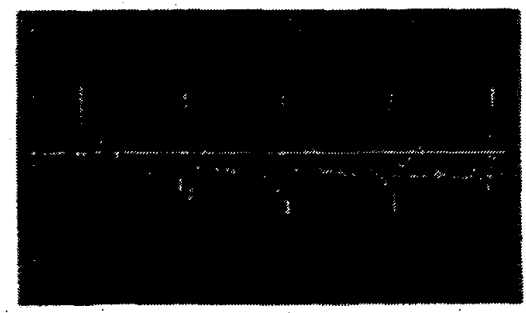

Figure 17. Normal ECG.

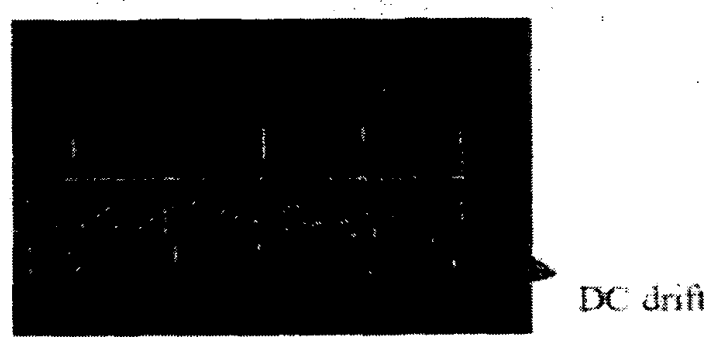

Figure 18. ECG with DC drift. 
As a result, an 8-bit ADC that can be accessed and addressed in a single process is carefully selected and tested. Another trade-off comes from that although we implemented some filters to enhance the quality of signals, these filters also distort the TDM signals (containing high frequency components). Thus we applied a software FIR digital filter to process the signals. Another obstacle comes from that we cannot get any pathological samples around. We solved this problem by testing the capability of measuring low oxygen saturation value by holding breath for $30 \sim 90$ seconds, and recorded the curve of relationship between oxygen saturation value and time. The curve in Fig. 19 shows a decreasing $\mathrm{SaO}_{2} \%$ when one keeps holding his breath for 75 seconds.

Finally, we roughly estimated the cost of the EVE system. In addition to the main expenses, the Bluetooth module and the development software, the remaining cost of EVE is practically little. Table VII shows the additional costs of our system. We can see that the EVE can be considered a low cost system.

TABLE VII

ADDITIONAL COSTS OF THE EVE SYSTEM.

(IN ADDITION TO BLUETOOTH MODULE AND DEVELOPMENT SOFTWARE)

\begin{tabular}{|c|r|}
\hline Item & \multicolumn{2}{|c|}{ Cost } \\
\hline $\begin{array}{c}\text { DS-100A } \\
\text { (Oximter Finger Probe) }\end{array}$ & $\$ 180.00$ \\
\hline GPS Module & $\$ 45.00$ \\
\hline Electrodes & $\$ 8.55$ \\
\hline The EVE Monitor & $\$ 20.00$ \\
\hline $\begin{array}{c}\text { Thermometer } \\
\text { (including AD590) }\end{array}$ & $\$ 6.00$ \\
\hline Other Passive Elements & $\$ 10.00$ \\
\hline \multicolumn{2}{|c|}{ Total } \\
& $\$ 269.55$ \\
\hline
\end{tabular}

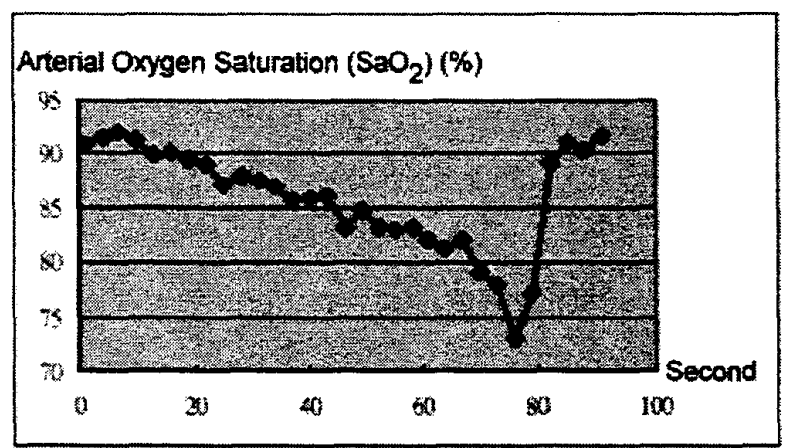

Figure 19. Expcriment result of breadth-holding test.

\section{CONCLUSIONS AND FUTURE WORK}

The E-Vanguard for Emergency is an easy-to-use system that helps save people's lives in hazardous conditions. We make the best use of the Bluetooth technology to construct a timely rescue wireless network with the following features. First, the implementation of the Scattemet enables the server to have efficient control over a large area for all the rescue operation. Second, the EVE Monitor Kit consists of a set of sensors (ECG, oximeters, and thermometers) that help detect and collect valuable physical conditions of the injured and the rescuers from the early stage of the rescue operation. Third, an integrated tracking system for outdoors has been constructed. This increases the management flexibility and enables an efficient resource allocation. Last, by means of the central server, the client (rescuers and medics), and the wireless techniques, the EVE can send out the patients' physical data as soon as possible for an early rescue preparation or decisionmaking.

In the future, the EVE system will be ported to a variety of rescue operations, such as the rescue of mountain disasters and homecare for patients with chronic illness. In addition, the size of the sensor module can be further reduced to be watch-sized for convenience. Any new sensor such as a scaffold-collapse detection sensor, which measures the deformation of pillars, and a hemadynamometer can be also added into the EVE Monitor kit.

\section{ACKNOWLEDGMENT}

The authors thank National Taiwan University Hospital and Taiwan Fire Administration for many helpful discussions and comments.

\section{REFERENCES}

[1] Bluetooth Specification Version 1.1, Bluetooth SIG, Feb. $22,2001$.

[2] Godfrey Tan, Allen Miu, John Guttag, and Hari Balakrishnan, "Forming Scatternets from Bluetooth Personal Area Networks," MIT-LCS-TR-826, Oct. 2001.

[3] Tree Scatternet Formation Algorithm, The Blueware Project, MIT Lab for Computer Science.

[4] Microsoft Visual Studio .Net Help.

[5] I. Yoshiya, Y. Shimada, and K. Tanaka, "Spectrophotometric Monitoring of Arterial Oxygen Saturation in the Fingertip," Med. \& Biol. Eng. \& Comput., vol. 18, 1980, pp. 27-32.

[6] J. P. De Kock and L. Tarassenko, "Pulse Oximetry: Theoretical and Experimental Models," Med. \& Biol. Eng. \& Comput., vol. 36, 1989, pp. 625-628.

[7] Nan-Shen Yeh, "Monitoring of Respiratory Rhythm and Oxygen Saturation in the Fingertip," Bulletin of the College of Engineering, NTU, no. 70, June 1997, pp. 115-125.

[8] Private Communication with Yuan-Hsiang Lin and L.P.Yamg. 